\title{
DETRÁS DEL TELÓN DE LA ENTREVISTA, LAS SITUACIONES QUE NOS INTERPELAN EN EL TRABAJO DE CAMPO ETNOBIOLÓGICO Y NO SE PLASMAN EN EL TEXTO
}

\author{
BEHIND THE INTERVIEW CURTAIN, GENDER RELATIONS IN \\ ETHNOBIOLOGICAL FIELDWORIK \\ Daily GARCÍA $^{1}$, Violeta FURLAN $^{1,2}$, Jessica MANZANO GARCÍA ${ }^{2}$, María Luján AHUMADA ${ }^{3}$ \\ ${ }^{1}$ FCF-UNaM, CeIBA, IBS-CONICET, ${ }^{2}$ Idacor, Museo de Antropología, FFyH, UNC, CONICET, ${ }^{3}$ Est. Forestal-INTA Villa Dolores, \\ CONICET \\ *dailysof@gmail.com
}

Submitted: 10/08/2020; Accepted: 02/12/2020; Published: 27/02/2021

\section{RESUMEN}

El trabajo presenta los desarrollos y debates planteados a partir de un taller realizado en el marco de las II Jornadas Argentinas de Etnobiología y Sociedad. Se visibilizó de manera colectiva diferentes situaciones vivenciales, experimentadas durante actividades de trabajo de campo etnobiológico y dentro de la filial institucional. Bajo diferentes estrategias metodológicas se estimularon habilidades visoperceptivas a través de frases que coaccionan en el ámbito laboral, propuestas por las coordinadoras de la mesa incentivando a la narración de sucesos atravesados por violencia. Participaron 31 personas, quienes debatieron y generaron desde sus perspectivas las posibles formas de accionar ante casos emergidos, tanto en las comunidades estudiadas como en la academia. A partir del análisis de las situaciones vividas y los marcos legislativos argentinos se construyó una lista de alternativas y recaudos. Grupalmente coincidieron en varios puntos tales como la escucha sin juzgamientos, la facilitación de información a la víctima, la relevancia de la capacitación en la temática a nivel institucional y la generación de redes de apoyo. Las investigadoras participantes del taller contrastaron aspectos beneficiosos y negativos para las mujeres en ciencia, resultando positivo el reconocimiento de licencias por maternidad y un mayor acceso a cargos directivos. Sin embargo, se cuestiona la naturalización de actitudes machistas dentro del sistema. Finalmente se contó con la asesoría legal y jurídica de una profesional perteneciente al Polo de la Mujer (Córdoba), experta en problemáticas de violencia, ofreciendo un asesoramiento basado en una amplia gama de herramientas teórico-prácticas, incluyendo canales de comunicación para proceder ante circunstancias que interpelan laboralmente de manera directa e indirecta a las personas que trabajamos en el campo de la etnobiología. Se concluye la necesidad de promover espacios que desde un análisis situacional formulen y ejecuten de manera constante, estrategias de accionar preferiblemente desde un compromiso de capacitación e involucramiento institucional.

PALABRAS CLAVE: Comunicación, Red apoyo mutuo, Trabajo de campo etnobiológico, Violencia de género, Feminismos.

\begin{abstract}
The work presents the developments and reflections raised from a workshop carried out within the framework of the II Argentine Conference on Ethnobiology. It allowed to collectively visualize different situations, experienced during ethnobiological research in institutions and fieldwork.
\end{abstract}


Under different methodological strategies, proposed by the coordinators, visual-perceptual skills were stimulated through phrases that coerce in the workplace, in order to encourage the description of events crossed by violence. Thirty-one people participated, through group discussions they generated from their own perspectives the possible ways to act before emerging cases, both in the studied communities and in the institutional space. Based on the analysis of the situations experienced and the Argentine legislative frameworks, a list of alternatives and precautions was constructed. The groups agreed on various points such as listening without judgment, providing information to the victim, the relevance of training on the subject at institutional level, and the generation of support networks. The experienced researchers participating in the workshop contrasted beneficial and negative aspects for women in science, with the recognition of maternity leave and greater access to managerial positions being positive in the last years. However, they questioned the naturalization of sexist attitudes within the system. Finally, we had the legal advice of a professional belonging to the Polo de la Mujer (Córdoba), an expert in gender violence, offering advice based on a wide range of theoretical-practical tools, including communication channels to proceed under circumstances that directly and indirectly challenge the ethnobiologist. It concludes with the need to promote spaces that from a situational analysis formulate and execute constantly, action strategies preferably from a commitment to training and institutional involvement.

KEYWORDS: Communication, Mutual support net, Ethnobiological fieldwork, Gender violence Feminist theory.

\section{INTRODUCCIÓN: SE ABRE EL TELÓN}

La Etnobiología se orienta a poner en valor la diversidad de conocimientos en relación a los vínculos establecidos entre los seres humanos con el resto de los seres vivos (MEDRANO, 2014; LADIO, 2017). Dada su búsqueda acerca de los saberes múltiples o epistemes, la Etnobiología puede pensarse como una disciplina que pone de manifiesto la construcción de conocimientos a partir de mundos relacionales u ontologías relacionales (ESCOBAR, 2016), representados por densas redes de interrelaciones de todo tipo de seres y formas de vida, a través de una serie infinita de prácticas, mitos, creencias, leyendas, relatos, y donde todos los seres vivientes dependen de otros para su existencia (INGOLD, 2011). Esta forma de entender la etnobiología define diferentes situaciones, en particular, en este trabajo, nos centraremos en dos acercamientos y formas de entender la etnobiología que guían la práctica de la disciplina: 1- el acercamiento a los planteos de las luchas y discursos de las corrientes ecofeministas (LADIO, 2020), que buscan transformar las maneras en que las personas se relacionan entre sí y con la Naturaleza, eliminando las conocidas formas de opresión, imposición y apropiación instaladas en las visiones androcentristas (PULEO, 2009; RODRÍGUEZ y HERRERO LÓPEZ, 2010); 2- el alejamiento del pensamiento patriarcal, el cual estructura el mundo en pares opuestos contrarios entre sí, y de desigual valor, que separan y dividen la realidad, tal como el 
dualismo Naturaleza-Cultura (RODRÍGUEZ y HERRERO LÓPEZ, 2010; FURLAN et al., 2020).

Ahora bien, las metodologías de acercamiento a otros mundos para su conocimiento, puesta en valor y por consiguiente su visibilidad, han sido mediante el uso de técnicas etnográficas (LÓPEZ DEL POZO, 1992), a través de las cuales no solo se registran hechos, son momentos de encuentro, en donde se sientan posiciones, se intercambian ideas, se reflexiona sobre realidades y se construyen discursos (GUBER, 2004). También son momentos de exposición mutua, a veces de confrontación, que como los definiría Halperín (1995), aborda desde la intimidad del entrevistado, pensamientos, razones ocultas y debilidades, esbozando así el "arte del vínculo". Durante la construcción de ese vínculo, pueden surgir situaciones de violencia expresadas en la narración de los interlocutores o la observación del investigador. Sin embargo, en los contextos de violencia no bastan las destrezas comunicativas, se requieren herramientas teóricas y prácticas que faciliten en estos casos formas de intervención y toma de decisiones (JIMENO; VARELLA; CASTILLO, 2011). De esta manera, las personas que realizamos o intentamos realizar el trabajo de campo etnográfico con enfoque de género, encontramos al menos dos situaciones desafiantes que aquí expondremos. Por un lado, resignificar nuestras formaciones disciplinares para la construcción de herramientas que nos permitan comprender y describir la complejidad de las problemáticas que estudiamos en los diversos mundos (TRPIN et al., 2017) y, por otro lado, contar con herramientas que permitan accionar frente a patrones socioculturales de discriminación y violencia asociados al género.

Si bien existen en Argentina marcos normativos para accionar frente a estas situaciones en el contexto académico, tal como la Ley 26.485/2009 de Protección integral para prevenir, sancionar y erradicar la violencia contra las mujeres en los ámbitos en que desarrollen sus relaciones interpersonales, o la reciente Ley 27.499/2019 conocida como Ley Micaela de Capacitación, obligatoria en la temática de género y violencia contra las mujeres, su puesta en práctica e incorporación en las tareas de investigación tanto para el personal académico como para su aplicación en la población es compleja.

Dado que las situaciones de violencia también forman parte del mundo de la investigación, al menos en la tarea etnográfica como aquí exponemos, pretendemos expandir el debate acerca de nuestro accionar frente a dichas situaciones en la construcción de diversos mundos, dado que no sólo resulta importante el pleno reconocimiento del otro y sus vivencias, sino también contemplar nuestro posible proceder resolutivo (COVA JAIME, 2012). Sin dudas, esto conlleva a la descolonización de nuestras prácticas disciplinares, tal 
como afirma Segato (2010), lo que implica dar cuenta de ciertos desafíos que nos involucran en el campo para ampliar nuestras opciones teóricas y metodológicas; considerando que la propia reflexividad "al contrastarse con la de los sujetos que estudia; se resignifica y encuentra un nuevo lugar" (GUBER, 2004, p. 87).

En base a esta preocupación común, las autoras convocamos en el marco de las II Jornadas Argentinas de Etnobiología y Sociedad del mes de noviembre de 2019 a un taller con el fin de incentivar la participación activa de la comunidad etnobiológica a involucrarse desde su experiencia, a reflexionar sobre situaciones vividas ante casos de violencia de género, violencia intrafamiliar, prejuicios e incertidumbres dados en el contexto del trabajo etnobiológico. En particular, en instancias de trabajo de campo y los posibles accionares frente a las mencionadas situaciones. Consideramos que el enfoque etnográfico, fundamental en la construcción de la disciplina etnobiológica, puede convertirse en una forma de ejercicio de la ciudadanía (JIMENO; VARELA; CASTILLO, 2011). De esta manera, "Detrás del telón" es una invitación a la reflexión colectiva y generación de ciencia involucrada, de ciencia comprometida, siguiendo la línea de pensamiento propuesta por Segato (2010) desde la cátedra de pensamiento incómodo.

\section{MATERIALES Y MÉTODOS: ENTRE BAMBALINAS}

El taller se realizó en el mes de noviembre del año 2019, en el marco de las II Jornadas Argentinas de Etnobiología y Sociedad. Se convocó a quienes asistieron a las jornadas a participar independientemente de su género, edad y perfil profesional, esto con la intención de contar con una variedad de perspectivas, saberes, sentires y necesidades.

Las herramientas teórico-prácticas usadas en el taller se enmarcan en un proceso analítico, atravesado a su vez por contextos situacionales, que van demarcando el campo propio de la investigación cualitativa, pero esta vez dando lugar a modelos metodológicos no convencionales. Para ello, seguimos los lineamientos planteados por Denzin y Lincoln (2011), haciendo uso de estrategias de participación basada en representaciones conversacionales, críticas, visuales, construidas de manera colectiva y abierta a múltiples voces.

El espacio propuesto constó de la recolección de información cualitativa como mecanismo de indagación exploratoria, descriptiva y propositiva, con un grupo focal subdividido y las experiencias recopiladas fueron interpretadas a partir de Teoría Fundamentada (STRAUSS; CORBIN, 2002). 
El taller estuvo compuesto por cuatro formas de participación e interacción: estimulación visual, dinámica grupal, debate y reflexión.

\subsection{Estimulación visual}

Con el objetivo de estimular las habilidades visoperceptivas (atención, memoria y procesamiento) que permiten interpretar y comprender la información percibida y con el fin de lograr una participación activa, el debate se inició a partir de carteles que contenían frases que expresaban desigualdades de género así como de violencia intrínseca. Estos, por su vez, fueron situados en diferentes puntos del espacio utilizado, siendo disparadores de ideas o situaciones en torno a distintos escenarios de violencia.

\subsection{Dinámica grupal}

Después de un tiempo de lectura e interiorización de las frases, se procedió a la conformación de grupos de trabajo. Los equipos, debían plasmar en carteles tipo papelógrafo, conceptos, opiniones e intenciones que representaran un canal comunicativo y de acciones posibles, compuesto por elementos indispensables en el manejo de situaciones de violencia. Posteriormente se dio apertura a la socialización de las ideas colectivas.

\subsection{Debate}

El momento de puesta en común de la actividad grupal fue grabado y desgrabado de manera textual para su estudio. Se organizó la información de acuerdo con las categorías que representan diferentes puntos de análisis, las cuales surgieron como temáticas comunes entre diferentes participantes.

\subsection{Reflexión}

Finalmente, se contó con una asesoría profesional especializada en asesoramiento ante situaciones de violencia de género. La asesora explicó al grupo acerca de herramientas, de medidas de apoyo y mecanismos de visibilización o comunicación, fundamentales en el tratamiento de situaciones de violencia posibles, tanto de interpelación laboral o extralaborales. A modo de cierre, el debate grupal se orientó a la conclusión de un conjunto de preguntas que invitan al autoconocimiento y a la reflexión colectiva.

\section{RESULTADOS: EN LA ESCENA}


El taller tuvo una participación de $17.5 \%$ de los participantes de las II Jornadas de Etnobiología. Con un rango de edad desde los 22 a los 52 años, siendo en su mayoría mujeres, con participación de varones y disidencias.

Luego del análisis pormenorizado de la actividad de debates grupales y producción de afiches por grupo de trabajo, podemos dar cuenta que la totalidad de los participantes manifestó haber vivido o presenciado situaciones de violencia de género o intrafamiliar, durante sus tareas de campo en comunidades de diferentes regiones de Argentina. Situaciones vividas al menos una vez en zonas tanto urbanas como rurales. En barrios urbanos, con comunidades campesinas e indígenas sin importar el género de la persona investigadora involucrada, coincidiendo en aspectos perceptivos, emocionales y conductuales, subyacentes a las circunstancias. En este sentido recuperamos fragmentos narrativos compilados en los Datos Suplementarios de éste trabajo de algunas de las personas que intervinieron en la puesta en común de las actividades que resumimos a continuación.

\begin{tabular}{|c|c|}
\hline $\begin{array}{c}\text { Temáticas emergentes } \\
\text { agrupadas }\end{array}$ & Narrativas relacionadas \\
\hline $\begin{array}{c}\text { Percepciones, emociones y } \\
\text { conductas } \\
\text { asociadas a escenarios de } \\
\text { violencia }\end{array}$ & Posturas sin juzgamientos, el respeto y discreción; \\
\hline $\begin{array}{c}\text { Falta de capacitación y la } \\
\text { responsabilidad } \\
\text { institucional ante situaciones de } \\
\text { violencia }\end{array}$ & $\begin{array}{c}\text { Pronta detección, reconocimiento y accionar desde el } \\
\text { proceder "correcto" entre lo ideal y lo legal. }\end{array}$ \\
\hline $\begin{array}{c}\text { Reconocimiento de posición de } \\
\text { poder } \\
\text { desde nuestro lugar en la } \\
\text { academia }\end{array}$ & $\begin{array}{c}\text { Herramientas de cambios sociales ante escenarios de } \\
\text { violencia y no como formas de distanciamiento social. }\end{array}$ \\
\hline $\begin{array}{c}\text { Situaciones de violencia donde } \\
\text { la víctima es quien entrevista }\end{array}$ & $\begin{array}{c}\text { Comprender el sistema de relaciones sociales que } \\
\text { coloca en situación de riesgo y vulnerabilidad. } \\
\text { Búsqueda de apoyo y comunicación fluida en el equipo } \\
\text { de trabajo. }\end{array}$ \\
\hline $\begin{array}{c}\text { Percepciones desde el ámbito } \\
\text { académico }\end{array}$ & $\begin{array}{c}\text { Desigualdades adyacentes, acoso y abusos naturalizados } \\
\text { en muchas instituciones; }\end{array}$ \\
\hline
\end{tabular}

Tabla 1. Resumen del compendio de narrativas sobre las temáticas emergentes determinadas en el taller realizado. Fuente: Las autoras (2020).

\subsection{Reflexiones post-debate}

Entre las concordancias finales del taller, quienes participaron sugieren que se requiere de reformas no solo en términos legales, como parte de la emancipación y 
liberación de las situaciones de opresión y dominación referidas al género, sino de una integración de aspectos como los institucionales en la reestructuración de los cambios que implique una transformación cultural. La intención es disminuir los efectos nocivos del patriarcado sobre las personas y, como búsqueda a largo plazo, la generación de un modo de relacionarse que esté basado en la colaboración y respeto de los derechos inalienables de personas y organismos en general. A continuación, algunos fragmentos discursivos:

"El problema es el patriarcado, y el ejemplo que ella está poniendo es mujeres machistas, que también las hay.......actitudes machistas tenemos todas las personas en algún momento.... El hombre es quien históricamente ha tenido privilegios y nosotras no, y no necesariamente esos privilegios significan violencia, sino son cuestiones inconscientes, privilegios en torno hasta cómo usar el espacio común, que son muy sutiles y nosotras desde chicas hemos vivido situaciones de violencia o de inequidades, desigualdades, y ese es el punto. $Y$ es el patriarcado el problema, no son los hombres. Y hay hombres feministas también, pero todos estamos en un proceso de rever, de mover todo lo que hemos aprendido" (Participante A).

"A mi me parece que las maneras más rápidas y efectivas que pueden llegar a haber de cambios como los que estamos hablando acá, me parece que es establecer, poner en tela de juicio, llevar una discusión, llevar una especie de atención permanente y de denuncia $o$ de discusión permanente de situaciones que vemos en lo cotidiano, pero en las instituciones, o sea en la medida que vayan cambiando las instituciones, va a ir cambiando la sociedad también. Eso se transfiere, es casi mecánico. Porque funcionamos a través de instituciones" (Participante $\mathrm{B}$ ).

Además, el debate dio lugar al análisis, la introspección y repercutió de manera reflexiva en quienes participaron del taller.

"Si en verdad, son los dos procesos, de abajo hacia arriba y desde arriba hacia abajo, porque es la forma que va atravesando para mi la discusión, todos de acá se van a llevar algún ruido" (Participante C).

Dentro del abanico de intervenciones, aun siendo minoría hubo cavilaciones de la participación masculina, tal como la siguiente mención, relacionadas a situaciones de violencia de género dentro de las instituciones: 
"Ustedes están cansadas de hacer planteos y no les dan bolilla, entonces en ese caso capaz la estrategia sea salir a los medios de prensa de la localidad, denunciarlos y ponerlos en evidencia, en situación de vergüenza. Porque ya a esta altura de la discusión las instituciones entran en un estado de deuda con la sociedad" (Participante D).

Se retomó en la discusión de cierre la importancia de la existencia de instrumentos en los procesos de visibilización y capacitación sobre violencia y género, así lo define el siguiente comentario:

"De hecho, estamos hablando esto hoy porque hay un movimiento de mujeres y de feminismo que se está traduciendo en la Ley Micaela y que las instituciones ahora tienen que capacitarse. Tendrían que ir al mismo ritmo las instituciones, pero el movimiento de base va mucho más rápido, por suerte" (Participante E).

A continuación, la intervención de la mirada profesional y la invitación a continuar formando vínculos de trabajo y esfuerzos colaborativos que forjen nuestras intenciones de igualdad de género:

"He nacido en una sociedad machista y patriarcal, y debo deconstruirme, si tomo ese trabajo, y tomo ese esfuerzo, que es todo un desafio, podemos empezar a pensar que en nuestra sociedad hay esperanza de que se logre algún cambio. $Y$ sobre todo que esto, un análisis de que ya no es nunca más en compartimentos estancos, acá me gustaría nombrar a la Denise Najmanovich, que viene diciendo hace tiempo, fácilmente 20 años de que la realidad no es más sólida, no es blanco-negro, no es hombre-mujer, el ying-yang, la hetero-normatividad, lo malo-bueno, victima-victimario, de hecho, nosotras no usamos esa categorización" (Participante F).

"No pongo la propuesta de protocolo porque creo que tendríamos que charlarla entre todas y ver si la proponemos... capaz puede ser unida a la encuesta que hablamos o algo para ya cuando lo escribamos lo tengamos avanzado y podamos coordinar con futuros encuentros y trabajos..." (Participante G).

"Lo que hacemos desde el año 2015 (año en que surge el movimiento Ni Una Menos en Argentina) es ir todas las mujeres juntas del brazo, porque asi nos dimos cuenta que se puede salir de una situación de violencia. Y siempre se lo decimos, no es sola que se sale de las situaciones de violencia. Las estrategias para dañar, incluyen el aislamiento de la persona, para que no pueda hacer nada, entonces las estrategias son siempre al revés, en conjunto, 
asesorándose, en grupo, aunque yo tenga como pretexto: vengan a tejer. $Y$ ahi en manada las mujeres nos encontramos, seguimos hablando, de los prejuicios, de lo que él me dijo y me duele, o lo que ella me ha dicho y me duele. Creo que han pensado un montón de estrategias que está bueno continuar pensando más, que es la manera, juntos. Y ojalá continúen más talleres, por ahí más concentradito en tiempo para que habilite otras cosas" (Participante $\mathrm{H})$.

\section{DISCUSIÓN: DESENLACE}

Tal como lo menciona Ladio (2020) los estudios etnobiológicos lograron visibilizar valores emocionales en relación a plantas y animales. Desde nuestro aporte, proponemos ampliar estos valores de personas a personas como con su entorno, involucrando todas las categorías que les atraviesan, interpelan y definen. Así como Trpin et al. (2017) sostienen en situar a las y los investigadores en el mismo plano crítico que el objeto explícito de estudio, siendo parte de interpelaciones permanentes en el quehacer de la investigación, con el presente estudio consideramos que si nos involucramos éticamente en nuestro trabajo de investigación, las interpelaciones (que forman parte del proceso) debemos corresponderlas de la mejor forma posible, con herramientas tangibles dentro del sistema de investigación actual y que al mismo tiempo se logre tomar en cuenta esta perspectiva en los trabajos y en las instituciones de investigación.

Las situaciones vividas en el campo que han quedado latentes en nuestras mentes más allá del trabajo explícito de investigación-, es lo que Fals Borda (2009) y Escobar (2016) consideran como el sentipensar del territorio. De acuerdo con estos autores, consideramos de gran importancia las manifestaciones emocionales tomando en cuenta la idiosincrasia de cada lugar, donde esa observación participativa sea con tendencia de real compromiso si se busca la conservación del paisaje en su totalidad.

\section{CONCLUSIÓN: FINAL DEL PRIMER ACTO}

Este aporte es un llamado a que cada uno en su lugar y contexto tomen el poder de trabajar con una mirada más ahusada de la conservación, de la Etnobiología, de las situaciones vividas y compartidas. Espacios donde podamos expresarnos y reflexionar con una mirada participativa y entender el sentido de las palabras de quienes nos interpelan. Esta apertura pretende tomar con mayor apego muchas desestructuras de nuestras costumbres ya que cada voz expuesta en el taller realizado, abrió el telón a la imperante necesidad de evaluar cambios de paradigma posibles, de evaluarlo desde el código de ética 
internacional de etnobiología (ISE, 2006; SOLAE, 2015) hacia nuestras instituciones de trabajo. Donde por ejemplo si hablamos de agroecología y manejo de remanentes de monte y exista un déficit de espacios para abordar temáticas emocionales y que al mismo tiempo la violencia se encuentre presente, no podríamos lograr el mantenimiento del bosque nativo, dejando de lado las situaciones de opresión en la localidad.

Entre varios puntos a seguir, se podría evaluar si muchas actividades de descuido de los paisajes naturales o el poco interés en conservación y apego a la naturaleza (como son la caza deportiva, la quema como modo de lucro, extractivismo, entre otras) se relacionan con una crianza en ambientes violentos y de explotación. Relaciones que no solo son domésticas, sino que se dan en el seno de la sociedad. Para que la ciencia pueda aportar en cambios de paradigmas en conservación y en etnobiología tiene que involucrarse y generar relaciones de respeto. En este trabajo decidimos profundizar en ese sentido, para así generar una nueva "escenografía” con los mismos actores y diferentes relaciones.

\section{AGRADECIMIENTOS}

Agradecemos a todas las personas participantes del taller, cada una desde su espacio y rol aportó puntos de discusión importantes para desarrollar una nueva forma de trabajar en etnobiología basada en el respeto mutuo y también en la interseccionalidad que nos encuentra en el trabajo de campo. Especialmente agradecemos a las mujeres y disidencias por seguir insistiendo en la importancia de este camino y a los varones por animarse a participar, escuchar, acompañar y dejarse interpelar por problemáticas que nos atraviesan a todas las personas.

\section{REFERENCIAS BIBLIOGRÁFICAS}

COVA JAIME, Y. La comprensión de la escucha. Letras [online]. Caracas, v. 54, n. 87, p. 98-109, 2012. Disponible en: http://ve.scielo.org/scielo.php?script=sci_arttext\&pid=S045912832012000200005\&lng=es\&nrm=iso. Accedido en: 23 dic. 2020.

DENZIN, N. K., LINCOLN, Y. S. El campo de la investigación cualitativa. Manual de Investigación Cualitativa Volumen I. Barcelona: Gedisa, 2011. 376 p.

ESCOBAR, A. Sentipensar con la Tierra: las luchas territoriales y la dimensión ontológica de las epistemologías del sur. AIBR: Revista de Antropología Iberoamericana, Madrid, vol. 11, n.1, p. 11-32, 2016. 
FALS BORDA, O. 1925-2008. Una sociología sentipensante para América Latina. Víctor Manuel Moncayo Compilador. Bogotá: Siglo del Hombre Editores y CLACSO, 2009.

FURLAN, V. et al. 'Ethnobiological equivocation' and other misunderstandings in the interpretation of natures, [S. 1.], Agosto 2020. Studies in History and Philosophy of Biol \& Biomed Sci. Disponible en: https://doi.org/10.1016/j.shpsc.2020.101333, Accedido en: 23 dic. 2020.

GUBER, R. El salvaje metropolitano: reconstrucción del conocimiento social en el trabajo de campo. Buenos Aires: Paidós, 2004. 325 p.

HALPERÍN J. La entrevista periodística: intimidades de la conversación pública. Paidos Iberica. Buenos Aires, 1995. 296 p.

INGOLD, T. Being alive: Essays on movement, knowledge and description. Taylor \& Francis, Reino Unido, $2011.270 \mathrm{p}$.

INTERNATIONAL SOCIETY OF ETHNOBIOLOGY (ISE). International Society of Ethnobiology Code of Ethics (with 2008 additions). 2006. Disponible en http://ethnobiology.net/code-of-ethics/. Accedido en: 23 dic. 2020.

JIMENO, M.; VARELA, D.; CASTILLO, A. Experiencias de violencia: etnografía y recomposición social en Colombia. Sociedade e cultura, Brasil, vol. 14, n.2, p.275-285, 2011. Disponible en: https://doi.org/10.5216/sec.v14i2.17604. Accedido en: 23 dic. 2020.

LADIO, A. H. Ethnobiology and research on Global Environmental Change: what distinctive contribution can we make? Ethnobiology and Conservation, [S. 1.], v. 6, 2017. Disponible en: https://www.ethnobioconservation.com/index.php/ebc/article/view/116. Accedido en: 23 dic. 2020.

LADIO A. Etnobiología en áreas rurales y su aporte a la lucha para desentrañar sesgos patriarcales. Ethnoscientia, [S.l.], jun. 2020. ISSN 2448-1998. Disponible en: http://www.ethnoscientia.com/index.php/revista/article/view/298. Accedido en: 23 dic. 2020. doi:http://dx.doi.org/10.22276/ethnoscientia.v5i1.298.

LÓPEZ DEL POZO, E. Etnobiología: síntesis teórica. América negra, vol. 3. 1992.

MEDRANO, C. Zoo-sociocosmología qom: seres humanos, animales y sus relaciones en el Gran Chaco. Journal de la Société des Américanistes, vol. 100, n.1, p. 233-265, 2014. Disponible en https://journals.openedition.org/jsa/13777, Accedido en: 28 dic. 2020. doi: https://doi.org/10.4000/jsa.13777

PULEO, et al. Claves del ecologismo social. España: Editorial Libros en AcciónEcologistas en Acción. 2009.

RODRÍGUEZ, M.; LÓPEZ, Y. Ecofeminismo, una propuesta para repensar el presente y construir el futuro. CIP-Ecosocial. Boletín ECOS, vol. 10, p. 1-3. 2010. Disponible en: http://na.attac.es/wpcontent/uploads/2016/06/ecofeminismo_propuesta_repensar_presente.p df. Accedido en: 28 dic. 2020. 
SEGATO; R. Género y Colonialidad: em busca de claves de lectura e de un vocabulario estratégico. Editorial Mimeo. México. 2010.

SOLAE, Sociedad Latinoamericana de Etnobiología. Código de Ética. Revista Etnobiología, 2016, vol. 14.

STRAUSS, A; CORBIN, J. Bases de la investigación cualitativa. Técnicas y procedimientos para desarrollar teoría fundamentada. Editorial Universidad de Antioquia. Medellín. 2002. 335p.

TRPIN, V.; RODRIGUEZ, M. D.; BROUCHOUD, S. Desafíos en el abordaje del trabajo rural en el norte de la Patagonia: Mujeres en forestación, horticultura y fruticultura. Trabajo y Sociedad, vol. 28, p. 267-280. 2016. Disponible en: https://ri.conicet.gov.ar/bitstream/handle/11336/65529/CONICET_Digital_Nro.b0b565e6cd95-46cf-9528-31b9b07345bd_A.pdf?sequence=2\&isAllowed=y. Accedido en: 28 dic. 2020 . 


\begin{abstract}
ANEXOS
Coincidiendo en aspectos perceptivos, emocionales y conductuales, subyacentes a las circunstancias. En este sentido recuperamos fragmentos narrativos compilados en los Anexos de algunas de las personas que intervinieron en la puesta en común de las actividades que resumimos en el siguiente cuadro.
\end{abstract}

\title{
Percepciones, emociones y conductas asociadas a escenarios de violencia
}

La valencia emocional con la que se sugiere a afrontar una situación de violencia desde una postura sin juzgamientos y el respeto por la decisión a proceder de la persona entrevistada, fueron menciones recurrentes entre los participantes del taller, así lo permiten entrever las siguientes frases textuales:

"Estuvimos viendo que todos y todas en algún momento vivenciamos o conocemos de situaciones de violencia de género, y que no sabemos cómo actuar, entonces pensamos que lo primero es siempre tratar de escuchar sin prejuicio, estar atentos, y como decía la compañera, ir sin que sea un prejuicio para no ser ingenuo frente a lo que uno puede encontrar. En caso de visualizar una situación de violencia o que alguien en el terreno o en una entrevista venga y cuente de una situación de violencia de género, tratar de brindar información, pero también ser cauteloso o cautelosa con lo que esa situación puede destapar".

"Manejar la información con discreción, no hacer un escándalo en la comunidad, no ser participe. No exponer a la víctima. Fijarse en las herramientas que existen, muchas son muy citadinas. Lo mismo que dijeron allá. Preguntarle a la víctima que quiere hacer, porque una le puede decir a la víctima que denuncie, y capaz que no quiere o no se siente preparada o cree que le va a pasar algo."

\section{Falta de capacitación y la responsabilidad institucional ante situaciones de violencia}

En general las personas que participaron del taller manifestaron haberse sentido involucradas presenciando en algún momento escenarios de violencia, asociándolos directamente con una reacción mental de tristeza como lo es la sensación de impotencia, al no poder controlar o solucionar la situación. Asimismo, la relevancia otorgada a la pronta 
detección, reconocimiento y accionar desde el proceder "correcto" o ideal, a partir de la instrucción normativa correspondiente frente a actos violentos, fueron otras de las convergencias discursivas entre quienes asistieron a la actividad, como se expresa a continuación.

"El tema de capacitarnos también lo vemos como fundamental, porque justamente no sabemos cómo actuar y podemos dejar pasar situaciones súper importantes por miedo o actuar de una forma que no sea la más adecuada por desconocimiento, también empezar a tejer redes. En 5 minutos vimos que todes vivimos estas situaciones y vimos que todes no sabemos cómo actuar, entonces poder hablar, no quedarnos callados frente a una situación que vivimos, personalmente que no nos quede como una anécdota de algo feo, de algo que no queremos volver a repetir y buscar espacio de contención y capacitación e ir tejiendo redes no sólo entre los que estamos en estos espacios privilegiados, sino también en el territorio."

"Me parece muy importante que cada una y cada une desde el lugar donde está conozca todo ese marco legal, cómo puede hacer, pero que también conozca el territorio, conozca a las personas, las situaciones, que trate de interiorizarse con los vínculos, porque por ahi una se arrebata y lo que una piensa que puede ser una solución, puede agravar el problema".

Del mismo modo e incluso con el nombramiento directo de instituciones, aludieron a la falta de herramientas instructivas y organizacionales para el manejo de dichas circunstancias, a través de menciones como la siguiente:

"Igual creo que como trabajadoras de la universidad, o de INTA o de CONICET, tenemos que demandar a nuestras instituciones que nos den formación para tener capacidades para manejar estas situaciones. Si nosotras no demandamos, nuestras instituciones hacen la vista ciega y oídos sordos a esto y nosotros estamos confrontándolo con buena voluntad, pero sin las herramientas apropiadas. Creo que es responsabilidad de las instituciones, bajo el marco legal de la Argentina acompañar tanto a los trabajadores varones como mujeres, darles las herramientas para que tengan la capacidad de actuar". 
Otra conjunción de ideas presentada, fue la necesidad de aplicación de la Ley Micaela, reconocida y citada entre algunos participantes, siendo este un mecanismo de capacitación obligatoria en género y violencia de género para todas las personas que se desempeñan en la función pública, en los poderes Ejecutivo, Legislativo y Judicial de la Nación. A continuación, menciones al respecto:

"Ahora con la aprobación de la Ley Micaela va a haber capacitaciones en todas las instituciones, capacitaciones obligatorias. Están empezando inclusive”

"Estoy como administrativa de una unidad, y ya empezaron las capacitaciones. Entonces tal vez como la figura de investigadora, investigador, becaria, becario si, la demanda debería existir."

También hubo un reconocimiento unánime de que estas situaciones no eran discutidas dentro de los propios espacios académicos, que poder hacerlo, así como darse cuenta que estamos expuestos a situaciones de violencia o ser interpelado por ellas es reciente.

Durante la sesión de taller, se presentaron consensos a travesados por emociones y conceptos de sincronía colectiva, que fueron elaborados a partir de criterios y dimensiones ligados a la significación de eventos experimentados individualmente. Así lo permitió entrever las herramientas cognitivo-conductuales sugeridas por los asistentes, quienes desde una postura empática proponían elementos considerados como fundamentales para la comprensión situacional, tales como la escucha, la atención y la consideración del otro para una posible intervención.

De esta manera fueron destacados múltiples aspectos recomendados, basados en la intuición, la búsqueda de ayuda profesional para manejar las situaciones con conocimiento, la formación de redes de contención, la instauración de denuncias ante el Estado en caso de menores de edad en situación de violencia y/o abandono, el acompañamiento para instituir denuncias en caso de personas mayores de edad, la búsqueda de apoyo en organizaciones locales con experticia en los temas y el reconocimiento de los estamentos del estado pertinentes. Además de establecer diferentes estrategias según el grado de violencia identificado. 
Por otro lado, usar el privilegio de formación académica para generar conexiones con otros profesionales que asistan los casos, comprender los tiempos y espacios de la persona en situación de violencia, que a su vez sugiere entender las diferentes aristas del problema a nivel local. Conjuntamente, fueron destacados elementos considerados como claves en la identificación y posible resolución de situaciones de violencia además de la escucha sin juicios, tales como la cautela, el mantener alerta, evitar ignorar contextos violentos y dispuestos a proporcionar ayuda.

\section{Reconocimiento de posición de poder desde nuestro lugar en la academia}

La diversidad epistemológica, ética y política son algunos de los matices sociales existentes entre quien entrevista y quien es entrevistado, dimensiones que suelen manifestarse a partir de una posición de poder por parte del entrevistador. Sin embargo, participantes del taller mencionaron lo útil que puede resultar la trayectoria académica como herramienta en el desarrollo factual como formas de cambios sociales a través de estrategias ante escenarios de violencia y no como formas de distanciamiento social. De tal modo se crean vínculos de horizontalidad y confianza que faciliten la comprensión entre personas. Así lo manifiesta una participante:

“Después también lo que planteábamos es que es importante tener presente que uno como persona, como investigador que va a un territorio diferente al nuestro, llevas una impronta de tener un cierto poder simbólico y que eso puede generar una cierta violencia si nosotros no sabemos interpretar los signos del resto. Entonces tener presente que cuando uno va a buscar información puede generar en el otro un rechazo, una violencia que estamos generando nosotros. Personalmente me parece bueno hacer hincapié en lo que dijeron las chicas, que si uno va al territorio es el hecho de estar, creo que con el tiempo uno puede introducirse más y generar una confianza donde te cuenten lo que está pasando”.

\section{Situaciones de violencia donde la víctima es quien entrevista}

Generalmente desde el conocimiento teórico científico y especialmente con el enfoque social de la etnobiología es necesario aprender a distinguir los grupos sociales a los que se vulneran sus derechos. En este caso específicamente nuestro interés apuntó inicialmente hacía a quienes entrevistamos y son víctimas de violencia. La finalidad radica en 
comprender el sistema de relaciones sociales que los coloca en situación de riesgo y a su vez en la condición de vulnerabilidad. Sin embargo, a medida que fuimos evocando experiencias, surgió que quienes entrevistamos también hemos sido blanco de violencia en diferentes niveles y contextos.

A partir de lo anterior, se expusieron estrategias básicas consideradas para quienes están trabajando en los territorios, entre las que se destacan la búsqueda de apoyo y la comunicación fluida en el equipo de trabajo, por ejemplo como estrategia para el conocimiento de movimientos individuales en el mismo.

\section{Percepciones desde el ámbito académico}

Se reconocieron las diferencias entre el pasado y el presente acerca de las licencias por maternidad que actualmente son reconocidas y antes estaban sujetas a la disposición de los directores o jefes. Es decir, las trabajadoras madres (en particular becarias o con cargos interinos) no solían contar con autorizaciones legítimas por parte de las instituciones, sino que eran gestos de "camaradería" laboral en los que se optaba por prescindir la ausencia de la persona.

Sin embargo, a pesar de la existencia actual de las licencias por maternidad, fueron manifestadas otras desigualdades adyacentes. La lógica evaluativa del sistema científico no contempla estos permisos, por lo que no se presenta una evaluación diferenciada en cuanto a concursos u objetivos logrados respecto de los propuestos.

Las investigadoras participantes también resaltaron la falta de acceso a altos cargos ejecutivos dentro de los sistemas de ciencia y tecnología de Argentina y Brasil.

Por otro lado, una docente e investigadora expresaba que el acoso generado por varones hacia mujeres está naturalizado en muchas instituciones. Que se hace visible cuando ese mismo acoso se da entre mujeres, pero no cuando esto se da desde varones a mujeres. Asimismo, se mencionaba que cuando una persona como mujer participa de espacios de poder espera y se prepara para enfrentar esos posibles abusos por parte de sus compañeros, sin distinción de género, varones con quienes tiene que tomar decisiones conjuntas. Por ejemplo, en juntas directivas o de evaluación de proyectos. 